\title{
Genetic code
}

\section{Enter a new amino acid}

\section{Dieter Söll}

BIOCHEMICAL processes in nature are characterized by the great variety of different compounds, yet only 20 amino acids take part in ribosome-mediated protein biosynthesis. Many non-standard amino acids, however, are found in a wide variety of proteins'. These 'additional' amino acids arise from post-translational modification of polypeptides synthesized by the established mechanism of protein biosynthesis. Recent evidence, however, indicates that the incorporation of selenocysteine into proteins is achieved in an unconventional way. The paper by Leinfelder et al. on page 723 of this issue ${ }^{2}$ provides a crucial detail for the mechanism of specific co-translational insertion of this unusual amino acid into the growing polypeptide chain in response to an in-frame UGA codon in the messenger RNA: there is a minor serine transfer (t)RNA species specific for selenocysteine capable of recognizing this UGA codon. Thus, the protein-synthesizing system is able to encode and use a twenty-first amino acid.

Selenium is a trace element in human diet. There are several enzymes in prokaryotic and eukaryotic cells which contain selenium in the form of selenocysteine in specific positions in the polypeptide chain ${ }^{3}$. This unusual amino acid is located in the active centre of some selenoenzymes (for example, Escherichia coli formate dehydrogenase and mammalian glutathione peroxidase) where the mostly ionized (at neutral $p \mathrm{H}$ ) selenol group is essential for the redox properties of these proteins ${ }^{3}$. The redox potential of the selenol group is about $100-150 \mathrm{mV}$ more negative than that of the corresponding thiol group of cysteine. This lower redox potential may be avantageous for the anaerobic conditions in which some of these enzymes are expressed and active.

The importance of selenocysteine is clear from studies of $E$. coli formate dehydrogenase: replacement of this amino acid by cysteine causes a 75 per cent reduction in enzyme activity, while replacement with serine renders the enzyme inactive (A. Böck, personal communication). In both $E$. coli formate dehydrogenase and human and murine glutathione peroxidase, selenocysteine incorporation is directed by an in-frame UGA nonsense codon $^{46}$. In vitro mutagenesis of the formate dehydrogenase gene demonstrates that selenocysteine incorporation is cotranslational ${ }^{7}$. To study the mechanism of this process, Leinfelder et al. collected several mutant $E$. coli strains impeded in the formation of formate dehydrogenase, and in this way identified four genes required for selenocysteine synthesis and for its incorporation into protein. As their paper in this issue ${ }^{2}$ shows, one of these genes encodes a minor tRNA ${ }^{\text {ser }}$ species. They therefore conclude that selenocysteine is formed by modification of serine aminoacylated to a natural suppressor tRNA capable of reading UGA.

These findings raise some interesting questions about protein synthesis: first, how is serine, attached to this tRNA, converted to selenocysteine? A possible intermediate could be the well-known mammalian phosphoseryl-tRNA ${ }^{8}$, which

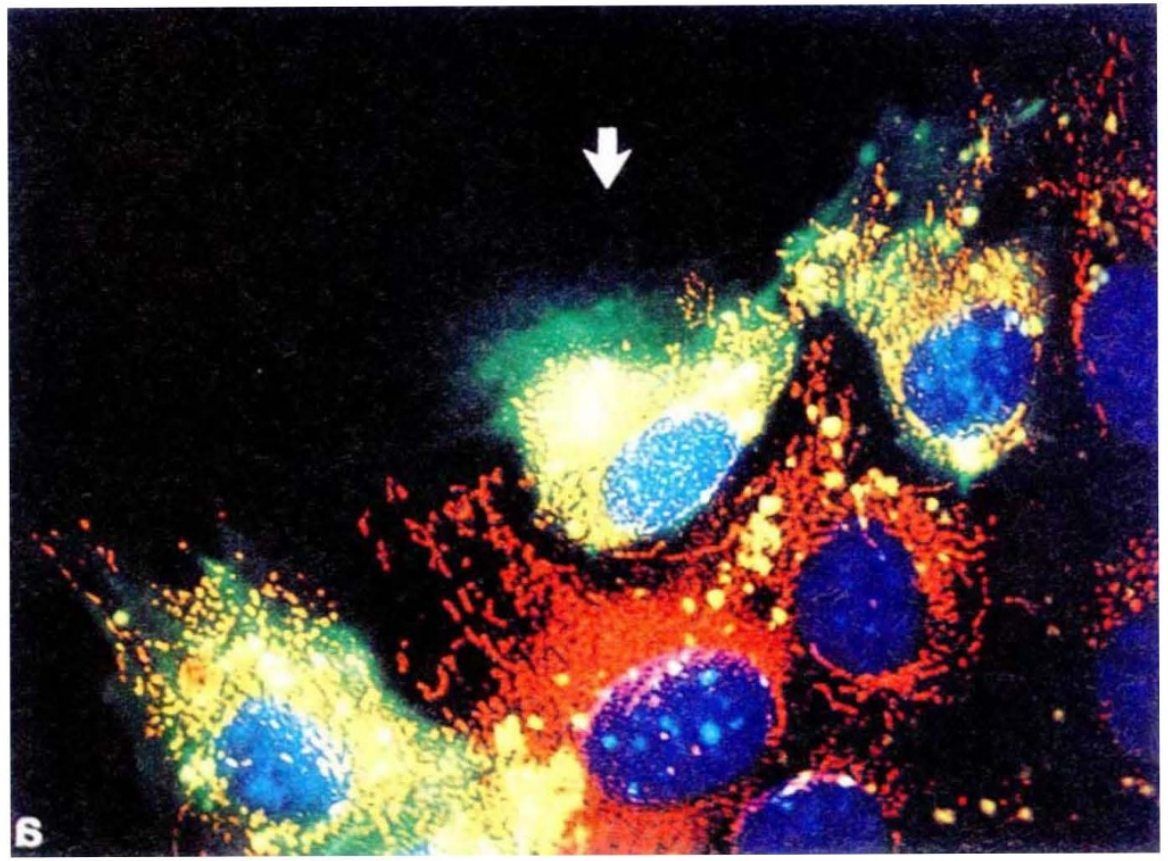

Robbin DeBiasio, Gary R. Bright, Lauren A. Ernst, Alan S. Waggoner and D. Lansing Taylor, in a recent issue of the Journal of Cell Biology $(105,1613-1622 ; 1987)$, demonstrate the combined use of different, specific fluorescent probes to elucidate how separate components of the cell are involved in different functions. Here, Swiss 3T3 fibroblast cells are shown migrating into a wound. Blue, cell nuclei; green, actin; red, mitochondria; yellow, endosomes. In their resting state, these cells do not have any detectable polarity of cell shape or particular distribution of organelles, and organize many of their actin filaments into stress fibres. In this micrograph, cells migrating into a wound are shown to become polarized, with the nucleus at the rear of the cell, the endosomes and mitochondria to the front side of the nucleus facing the direction of migration, and actin-based stress fibres at the rear of the cell. In the early stage of wound healing shown here ( 3 hours after wounding), the region directly behind the spreading lamellipodia (arrow) displays diffuse fluorescence, indicating that the actin filaments are not well organized. Later in the wound-healing process (not shown here, but see page 1,619 of the paper by DeBiasio et al.), both the endosomes and the mitochondria move into the lamellipodia and approach the leading edges of the cells. The cells become elongated and highly motile, and do not have detectable actin stress fibres. This method, combining fluorescence microscopy with the use of selective fluorescent probes, is a powerful approach for elucidation of chemical and structural changes in single, living cells during, for example, endocytosis, cell division and cell motility. 